\title{
ON BIALGEBRAS WHICH ARE SIMPLE HOPF MODULES
}

\author{
DAVID E. RADFORD ${ }^{1}$
}

\begin{abstract}
This paper gives a module characterization of commutative or cocom-
\end{abstract} mutative Hopf algebras over a field.

0. Introduction. Let $A$ be a bialgebra over a field $k$. Then $A$ has a natural left $A$-Hopf module structure, and if $A$ is a Hopf algebra, an easy calculation with the antipode shows that $A$ is a simple Hopf module. We show that a commutative or cocommutative bialgebra over a field $k$ which is a simple left Hopf module is a Hopf algebra. From this result we derive a module-theoretic characterization of commutative or cocommutative bialgebras over a field $k$ which are Hopf algebras; namely such a bialgebra is a Hopf algebra if and only if all left $\boldsymbol{A}$-Hopf modules are free (or (0)).

Generally a commutative or cocommutative bialgebra $A$ over a field $k$ has a unique maximal subcoalgebra $A_{(I)}$ which is a Hopf algebra. In both cases $A_{(I)}$ can be described in terms of grouplike elements-the basic results of this paper are derivatives of elementary observations concerning grouplikes in certain bialgebras.

The author wishes to thank the referee for his comments and suggestions, and wishes to express appreciation to Rutgers University for its hospitality during the time of the revision of this paper.

1. Preliminaries. In this section we show that any bialgebra $A$ over a field $k$ has a unique subcoalgebra $A_{(I)}$ maximal among the subcoalgebras $D$ such that the inclusion $i_{D} \in \operatorname{Hom}(D, A)$ has an inverse in the convolution algebra. We will see that $A_{(I)}$ is characterized by its simple subcoalgebras.

For a coalgebra $C$ over $k$ recall that the wedge product $U \wedge V$ of subspaces $U, V \subseteq C$ is defined by $U \wedge V=\Delta^{-1}(U \otimes C+C \otimes V)$. The wedge product of subcoalgebras is a subcoalgebra.

Lemma 1. Let $C$ be a coalgebra over a field $k$, and suppose $E, D^{\prime}, D^{\prime \prime} \subseteq C$ are subcoalgebras, $E$ simple.

(a) If $E \subseteq \sum D$, where $D$ runs over a family of subcoalgebras of $C$, then $E \subseteq D$ for some $D$.

(b) If $E \subseteq D^{\prime} \wedge D^{\prime \prime}$ then $E \subseteq D^{\prime}$ or $E \subseteq D^{\prime \prime}$.

Received by the editors July 18, 1979 and, in revised form, January 28, 1980.

AMS (MOS) subject classifications (1970). Primary 16A24; Secondary 16A48, 16A64.

$K e y$ words and phrases. Hopf algebra, bialgebra.

${ }^{1}$ Research for this paper was partially supported by NSF Grant No. MCS77-0013 A01. 
(c) If $A$ is a bialgebra and $E \subseteq D^{\prime} D^{\prime \prime}$, then $E \subseteq E^{\prime} E^{\prime \prime}$ where $E^{\prime} \subseteq D^{\prime}$ and $E^{\prime \prime} \subseteq D^{\prime \prime}$ are simple subcoalgebras.

(d) If $f: C^{\prime} \rightarrow C$ is a surjective coalgebra map, then $E \subseteq f\left(E^{\prime}\right)$ for some simple subcoalgebra $E^{\prime} \subseteq C^{\prime}$.

Proof. (a) is [S, Proposition 8.0.3.a]. To show (b) note that $\Delta E \subseteq D^{\prime} \otimes C+C$ $\otimes D^{\prime \prime}$ means $(E \otimes E) \cap\left(D^{\prime} \otimes C+C \otimes D^{\prime \prime}\right) \neq(0)$. By (a) if $U$ is a simple subcoalgebra of this intersection, then $U \subseteq D^{\prime} \otimes C$ or $U \subseteq C \otimes D^{\prime \prime}$, so $E \subseteq D^{\prime}$ or $E \subseteq D^{\prime \prime}$. To show (d) observe that $C_{0} \subseteq f\left(C_{0}^{\prime}\right)$ by [HR, 2.3.9]. Thus writing $C_{0}^{\prime}=\Sigma E^{\prime}$ as the (direct) sum of the simple subcoalgebras of $C^{\prime}$, we have $E \subseteq$ $\sum f\left(E^{\prime}\right)$, and hence (d) follows by (a). To show (c), first observe that $E \subseteq m(U)$ for some simple subcoalgebra $U \subseteq D^{\prime} \otimes D^{\prime \prime}$ by (d), where $m: D^{\prime} \otimes D^{\prime \prime} \rightarrow D^{\prime} D^{\prime \prime}$ is multiplication. By [HR, 2.3.13] $\left(D^{\prime} \otimes D^{\prime \prime}\right)_{0} \subseteq D_{0}^{\prime} \otimes D_{0}^{\prime \prime}$, so $U \subseteq E^{\prime} \otimes E^{\prime \prime}$ for simple subcoalgebras $E^{\prime} \subseteq D^{\prime}$ and $E^{\prime \prime} \subseteq D^{\prime \prime}$ by (a). Thus $E \subseteq E^{\prime} E^{\prime \prime}$. Q.E.D.

For a coalgebra $C$ and an algebra $A$ over $k$ recall that the unity of the convolution algebra $\operatorname{Hom}(C, A)$ is given by $\varepsilon(c)=\varepsilon(c) 1_{A}$ and the product is $f * g(c)=\sum f\left(c_{(1)}\right) g\left(c_{(2)}\right)$ for $c \in C$ and $f, g \in \operatorname{Hom}(C, A)$. The following is a refinement of [T, Lemma 14].

LEMMA 2. Let $C$ be a coalgebra and $A$ be an algebra over a field $k$, and let $x \in \operatorname{Hom}(C, A)$.

(a) If $x \equiv 0$ on $C_{0}$ then $X \mapsto x$ determines an algebra map $k[[X]] \mapsto \operatorname{Hom}(C, A)$. (Thus $x$ is invertible if $x \equiv \varepsilon$ on $C_{0}$.)

(b) $x$ is left (resp. right) invertible if and only if $\left.x\right|_{E} \in \operatorname{Hom}(E, A)$ is left (resp. right) invertible for all simple subcoalebras $E \subseteq C$. (Hence $x$ is invertible if and only if $\left.x\right|_{E}$ is invertible for all simple subcoalgebras $E \subseteq C$.)

Proof. (a) The $C_{n}$ 's form a filtration of $C$. Thus if $x \equiv 0$ on $C_{0}$, then $x^{n+1} \equiv 0$ on $C_{n}$ for $n \geqslant 0$ by induction, and therefore $\sum_{n=0}^{\infty} \alpha_{n} x^{n}$ is meaningful for all $\alpha_{0}, \alpha_{1}, \ldots \in k$. That $X \mapsto x$ extends to an algebra map is easy to check. If $x \equiv \varepsilon$ on $C_{0}$, we have just shown that $X \mapsto \varepsilon-x$ determines an algebra map, so $x=\varepsilon-(\varepsilon-x)$ is invertible since $1-X \in k[[X]]$ is.

(b) If $x$ is left invertible, then $\left.x\right|_{D}$ is for any subcoalgebra $D \subseteq C$. On the other hand, if $\left.x\right|_{E}$ is left invertible for all simple subcoalgebras $E \subseteq C$, then $\left.x\right|_{c_{0}}$ has a left inverse $f \in \operatorname{Hom}\left(C_{0}, A\right)$ since $C_{0}$ is a direct sum of simples. Let $F: C \rightarrow A$ be a linear extension of $f$. Then $F * x \equiv \varepsilon$ on $C_{0}$ which means $F * x$ is invertible by (a), hence $x$ is left invertible. The rest easily follows. Q.E.D.

Proposition 1. Let $C$ be a coalgebra and $A$ be an algebra over a field $k$, and let $f \in \operatorname{Hom}(C, A)$.

(a) Let $L_{(f)} \subseteq C$ (resp. $R_{(f)} \subseteq C$ ) be the sum of all subcoalgebras $D \subseteq C$ such that $\left.f\right|_{D}$ is left (resp. right) invertible. Then $\left.f\right|_{L_{(\Omega)}}$ is left invertible and $\left.f\right|_{R_{(\Omega)}}$ is right invertible.

(b) $C_{(f)}=L_{(f)} \cap R_{(f)}$ is the sum of all subcoalgebras $D \subseteq C$ such that $\left.f\right|_{D}$ is invertible, and $\left.f\right|_{C_{(\Omega)}}$ is invertible.

(c) $L_{(f)}$ and $R_{(f)}\left(\right.$ hence $\left.C_{(f)}\right)$ are closed under wedging. 
(d) If $\Omega$ is a field extension of $k$ then $L_{(f)} \otimes_{k} \Omega \subseteq L_{\left(f \otimes I_{0}\right)}$ and $R_{(f)} \otimes_{k} \Omega \subseteq$ $R_{\left(f \otimes I_{\Omega}\right)}$ (hence $C_{(f)} \otimes_{k} \Omega \subseteq C_{\left(f \otimes I_{\Omega}\right)}$ ).

(e) If $A$ is a bialgebra and $f$ is a coalgebra homomorphism, then $\left.f\right|_{C_{(\cap)}} ^{-1}$ is a coalgebra antihomomorphism.

Proof. (a) follows by Lemmas 2(b) and 1(a). (b) follows directly from (a). That $L_{(f)}$ and $R_{(f)}$ are closed under wedging, or equivalently, $L_{(f)} \wedge L_{(f)} \subseteq L_{(f)}$ and $R_{(f)} \wedge R_{(f)} \subseteq R_{(f)}$, follows by Lemmas 2(b) and 1(b). (d) is straightforward. The proof of [HS, 1.5.2.(6)] generalizes to a proof of (e). Q.E.D.

Let $A$ be a bialgebra over $k$ and let $L_{(I)}, R_{(I)}$ and $A_{(I)}$ denote the subcoalgebras of $C=A$ described in Proposition 1 for the identity map $I$ of $A$. One should note that $G\left(A_{(I)}\right)$ consists of all invertible $g \in G(A)$ and is therefore a group. (For a coalgebra $C$ over $k$ recall that $g \in C$ is grouplike if $g \neq 0$ and $\Delta g=g \otimes g$, and that $G(C)$ denotes the set of grouplike elements of $C$.) By Proposition 1(e) $s=\left(\left.I\right|_{A_{(I)}}\right)^{-1}$ is a coalgebra antihomomorphism. Observe that $s(g)=g^{-1}$ for $g \in G\left(A_{(I)}\right)$.

Suppose that $U$ and $V$ are vector spaces over $k$. For a field extension $\Omega$ of $k$ $\left(U \otimes_{k} \Omega\right) \otimes_{k}\left(V \otimes_{k} \Omega\right) \simeq\left(U \otimes_{k} V\right) \otimes_{k} \Omega((u \otimes \alpha) \otimes(v \otimes \beta) \mapsto(u \otimes v) \otimes \alpha \beta)$ is an isomorphism of $\Omega$-spaces. The Galois group $G(\Omega \backslash k)$ acts on $U \otimes_{k} \Omega$ as $k$-automorphisms by the rule $\sigma \cdot(u \otimes \alpha)=u \otimes \sigma \alpha$. (Thus $G(\Omega \backslash k)$ acts as $k$-algebra automorphisms if $U$ is an algebra.)

LEMMA 3. Let $C$ be a coalgebra over a field $k$ and suppose $\Omega$ is a field extension of k. Then $G\left(C \otimes_{k} \Omega\right)$ is a $G(\Omega \backslash k)$-module, which is cyclic if $C$ is cocommutative and simple and $\Omega$ is an algebraic closure of $k$.

Proof. If $g=\Sigma_{i} c_{i} \otimes \alpha_{i} \in C \otimes_{k} \Omega$, then $\Delta g=g \otimes g$ if and only if $\Sigma_{i} \Delta c_{i} \otimes \alpha_{i}$ $=\Sigma_{i j} c_{i} \otimes c_{j} \otimes \alpha_{i} \alpha_{j}$. From this it follows that $G\left(C \otimes_{k} \Omega\right)$ is a $G(\Omega \backslash k)$-module. Now assume $C$ is cocommutative and simple, and $\Omega$ is an algebraic closure of $k$. The isomorphism $C \otimes_{k} \Omega \simeq \operatorname{Hom}_{k}\left(C^{*}, \Omega\right)\left(c \otimes \alpha\left(c^{*}\right)=c^{*}(c) \alpha\right)$ restricts to an identification of $G(\Omega \backslash k)$-modules $G\left(C \otimes_{k} \Omega\right) \simeq \operatorname{Alg}_{k}\left(C^{*}, \Omega\right)$. Since $C^{*}$ is a finitedimensional field extension of $k$ and $\Omega$ is an algebraic closure of $k$, given $\tau, \tau^{\prime} \in \operatorname{Alg}_{k}\left(C^{*}, \Omega\right)$ there exists a $\sigma \in G(\Omega \backslash k)$ such that $\tau^{\prime}=\sigma \circ \tau$, i.e. $\operatorname{Alg}_{k}\left(C^{*}, \Omega\right)$ is cyclic. Q.E.D.

Let $V$ be a left $C$-module with basis $v_{1}, \ldots, v_{n}$. Define $e_{i j} \in C(1<i, j<n)$ by $\omega\left(v_{i}\right)=\sum_{j=1}^{n} e_{i j} \otimes v_{j}$. Then $\Delta e_{i j}=\Sigma_{k} e_{i k} \otimes e_{k j}$ and $\varepsilon\left(e_{i j}\right)=\delta_{i j}$ follow from the comodule axioms. If $C$ is a commutative bialgebra then the determinant $d=$ $\operatorname{det}\left(e_{i j}\right)$ of $\left(e_{i j}\right) \in M(n, C)$ is grouplike and does not depend on the choice of basis. Here we set $d_{V}=d$.

Let $C(n, k)$ be the coalgebra over $k$ with basis of symbols $e_{i j}(1<i, j<n)$ with structure defined as above. Let $V \subseteq C(n, k)$ have basis $e_{11}, \ldots, e_{n 1}$ and let $S(C(n, k))$ be the free commutative bialgebra on $C(n, k)$. Then $\mathscr{Q}_{n}(k)=$ $S(C(n, k))\left[d_{V}^{-1}\right]$ is a Hopf algebra since it represents the affine group scheme $\mathrm{GL}_{n}($ ) over $k$. 
2. The main results. Here we examine the role of grouplikes in certain bialgebras.

Proposition 2. Let $A$ be a bialgebra over a field $k$ which is commutative or has cocommutative coradical. Then $A_{(I)}$ is a Hopf algebra.

Proof. Assume $A$ is commutative. We will use Nichols' result [N] that a bialgebra quotient of a commutative Hopf algebra is a Hopf algebra. First let $V \subseteq A_{(I)}$ be a simple subcoalgebra and choose $e_{i j} \in A(1<i, j<n)$ for $V$ as indicated above. Since $V$ is a coalgebra, the $e_{i j}$ 's span $V$. From the equations $\Sigma_{k} e_{i k} s\left(e_{k j}\right)=\delta_{i j} 1$ we conclude that $\left(e_{i j}\right) \in M(n, A)$ is invertible, hence $d_{V}$ is invertible. Clearly $B_{V}=(V)\left[d_{V}^{-1}\right]$ is a bialgebra quotient of $\mathscr{U}_{n}(k)$, so $B_{V}$ is a Hopf algebra. In particular $s(V) \subseteq A_{(I)}$. For simple subcoalgebras $E^{\prime}, E^{\prime \prime} \subseteq A_{(I)}$ we apply Nichols' result again to multiplication $B_{E^{\prime}} \otimes B_{E^{\prime \prime}} \rightarrow B_{E^{\prime}} B_{E^{\prime \prime}}$ to deduce $E^{\prime} E^{\prime \prime}$ $\subseteq A_{(I)}$. Thus $A_{(I)}$ is a Hopf algebra by Lemmas 2(b) and 1(c).

Now assume $A_{0}$ is cocommutative and $\Omega$ is an algebraic closure of $k$. Then $A \otimes_{k} \Omega$ is pointed. $D=A_{(I)}+s\left(A_{(I)}\right)$ is a subcoalgebra of $A$ by Proposition 1(e). Note $G\left(s\left(A_{(I)}\right) \otimes_{k} \Omega\right)=G\left(s \otimes I\left(A_{(I)} \otimes_{k} \Omega\right)\right)=G\left(A_{(I)} \otimes_{k} \Omega\right)^{-1}$ by Lemma $1(\mathrm{~d})$, so $G\left(D \otimes_{k} \Omega\right)=G\left(D \otimes_{k} \Omega\right)^{-1}$ by part (a) of the same lemma. Thus by Lemma 1(c) the grouplikes of $(D) \otimes_{k} \Omega=\left(D \otimes_{k} \Omega\right)$ form a group. Therefore $(D) \otimes_{k} \Omega$ is a Hopf algebra by Lemma $2(\mathrm{~b})$, and this means $(D)$ is a Hopf algebra also. By definition $A_{(I)}=(D)$. Q.E.D.

Using Lemma 3 we have as a corollary to the proof:

Corollary 1. Let $A$ be a bialgebra over a field $k$, and suppose $C \subseteq A$ is a simple subcoalgebra.

(a) If $A$ is commutative, then $C \subseteq A_{(I)}$ if and only if $d_{C}$ is invertible in $A$.

(b) If $A_{0}$ is cocommutative and $\Omega$ is an algebraic closure of $k$, then $C \subseteq A_{(I)}$ if and only if some $g \in G\left(C \otimes_{k} \Omega\right)$ is invertible in $A \otimes_{k} \Omega$.

$A_{(I)}$ need not be a Hopf algebra in general.

EXAmple 1. Let $C=C(n, k) \oplus \mathbf{C}(n, k)$ and $T(C)$ be the free bialgebra on $C$. The ideal $I \subseteq T(C)$ generated by the relations described in $M(n, T(C))$ by $\left(e_{i j}\right)\left(e_{i j}\right)^{t}$ $=I=\left(\mathbf{e}_{i j}\right)^{t}\left(e_{i j}\right)$ is a bi-ideal. By the method of $\S 1$ of [B] one can show that $C \subseteq A \equiv T(C) / I$ (so $\left.C(n, k) \subseteq A_{(I)}\right)$ and that $\left(\mathbf{e}_{i j}\right) \in M(n, A)$ is not invertible (so $\left.s(C(n, k))=\mathbf{C}(n, k) \notin A_{(I)}\right)$ for $n \geqslant 2$.

Proposition 3. Let $A$ be a bialgebra over a field $k$ and suppose $C, C^{\prime} \subseteq A$ are nonzero subcoalgebras such that $C C^{\prime} \subseteq A_{(I)} \supseteq C^{\prime} C$. Then $C, C^{\prime} \subseteq A_{(I)}$.

Proof. Assume $C C^{\prime} \subseteq A_{(I)}$ and choose $a^{\prime} \in C^{\prime}$ such that $\varepsilon\left(a^{\prime}\right)=1$. Then $t \in \operatorname{Hom}(C, A)$ defined by $t(c)=\sum a_{(1)}^{\prime} s\left(c a_{(2)}^{\prime}\right)$ is a right inverse for $\left.I\right|_{C}$, so $C \subseteq$ $R_{(I)}$. Likewise $C^{\prime} \subseteq L_{(I)}$. Q.E.D.

Let $A$ be a bialgebra with left antipode $s$ (a left inverse of $I \in \operatorname{Hom}(A, A)$ ). Such an $A$ is a left Hopf algebra. Suppose $V \subseteq A$ is a left Hopf submodule (i.e. $\Delta V \subseteq A \otimes V$ and $A V \subseteq V)$. For $v \in V$ the calculation $\varepsilon(v) 1=\Sigma s\left(v_{(1)}\right) v_{(2)} \in A V$ $=V$ shows $V=(0)$ or $V=A$, so $A$ is a simple left $A$-Hopf module. 
THEOREM 1. Let $A$ be a bialgebra over a field $k$ which is commutative or has cocommutative coradical. Then the following are equivalent.

(a) $A$ is a Hopf algebra.

(b) $A$ is a simple left $A-H o p f$ module.

(c) If $C \subseteq A$ is a simple subcoalgebra then $A C=A$.

Proof. We need only show (c) $\Rightarrow$ (a). Assume (c) holds and let $C \subseteq A$ be simple. By Lemma 2(b) we need only show $C \subseteq A_{(I)}$. If $A$ is commutative, $d_{C}$ is invertible, so $C \subseteq A_{(I)}$ by Corollary 1(a). Suppose $A_{0}$ is cocommutative and $\Omega$ is an algebraic closure of $k$. Then $A C=A$ means $1 \in C^{\prime} C$ for some simple $C^{\prime}$ by Lemma 1(c), so $1=g^{\prime} g$ where $g^{\prime} \in G\left(C^{\prime} \otimes_{k} \Omega\right)$ and $g \in G\left(C \otimes_{k} \Omega\right)$ by the same result. Now replacing $C$ by $C^{\prime}$ we see $1=h^{\prime \prime} h^{\prime}$ for some $h^{\prime} \in G\left(C^{\prime} \otimes \Omega\right)$. P $g^{\prime}=\sigma \cdot h^{\prime}$ for some $\sigma \in G(\Omega \backslash k)$. Thus the calculation $1=\left(\sigma \cdot h^{\prime \prime}\right)\left(\sigma \cdot h^{\prime}\right)$ shows that $g^{\prime}$ is invertible, so $g$ is also, and $C \subseteq A_{(I)}$ by Corollary 1(b). Q.E.D.

A bialgebra which is a simple left Hopf module may not be a Hopf algebra (there exist left Hopf algebras which are not Hopf algebras [GNT]).

As a consequence of Lemma 2(b) and Corollary 1(a) a commutative bialgebra is a Hopf algebra if and only if all grouplikes are invertible [T, Corollary 69]. Generally this is not the case.

EXAMPLE 2. Let $C$ be a coalgebra over a field $k$ and let $T(C)$ be the free bialgebra on $C$. Then $T(C)^{n}=C \otimes \cdots \otimes C$ (n-times) has the tensor product coalgebra structure $(n>1)$. From the general coalgebra fact that

$$
G\left(C_{1} \otimes \cdots \otimes C_{n}\right)=G\left(C_{1}\right) \times \cdots \times G\left(C_{n}\right)
$$

and Lemma 1 (a) we conclude that $G(T(C))=\{1\}$ if $G(C)=\varnothing$. For example let $k=\mathbf{R}$ and $C=\mathbf{C}^{*}$. Then $T(C)$ is a cocommutative bialgebra, but is not a Hopf algebra, since $T(C) \otimes_{\mathrm{R}} \mathrm{C} \simeq T\left(C \otimes_{\mathrm{R}} \mathrm{C}\right)$ is the free monoid on $G\left(C \otimes_{\mathrm{R}} \mathrm{C}\right)$.

EXAMPLE 3. Let $C$ be a coalgebra which is also an algebra (possibly without unity), and suppose $\Delta, \varepsilon$ are multiplicative. The coalgebia structure of $C$ extends (uniquely) to a bialgebra structure on the algebra $A=k \cdot 1+C$ obtained by adjoining a unity to $C$. $A$ is not a Hopf algebra, since $C$ is a sub-Hopf module, unless $C=(0)$. For finite-dimensional examples where $G(A)=\{1\}$ let $C=C(n, k)$ and $e_{i j} e_{k l}=\delta_{i j} e_{k l}$.

Our last result gives a module-theoretic characterization of commutative or cocommutative Hopf algebras.

THEOREM 2. Let $A$ be a bialgebra over a field $k$ which is commutative or has cocommutative coradical. Then $A$ is a Hopf algebra if and only if all left A-Hopf modules are free (or (0)).

Proof. If $A$ is any Hopf algebra over a field, then all left Hopf modules are free (or (0)) by [S, Theorem 4.1.1]. Conversely, suppose $A$ satisfies this condition and let $C \subseteq A$ be a simple subcoalgebra. By Theorem 1 we must show $A C=A$, or $A / A C=(0)$. Since $A C$ and $A / A C$ are Hopf modules, $A C$ is free and $A / A C$ is free or (0). Since ker $\varepsilon$ is a codimension 1 ideal, any two bases of a free $A$-module $M$ 
have the same cardinality $r(M)$. If $A / A C$ is free, then $1=r(A)=r(A C)+$ $r(A / A C)$, a contradiction, so $A / A C=(0)$. Q.E.D.

"Free" cannot be replaced by "projective" in the preceding theorem since there are semisimple bialgebras which are not Hopf algebras. We close with a general construction.

EXAMPLE 4. Let $\mathscr{U}$ be an associative algebra (with unity) over $k$ with an algebra map $\delta: U \rightarrow U \otimes U$ satisfying $I \otimes \delta \circ \delta=\delta \otimes I \circ \delta$. The direct sum of algebras $A=k \cdot d \oplus \mathscr{Q}\left(d^{2}=d \neq 0\right)$ has a bialgebra structure determined by $\Delta d=$ $d \otimes d$ and $\Delta a=a \otimes d+d \otimes a+\delta a$ for $a \in \mathcal{Q} . A$ is a Hopf algebra if and only if $\mathscr{U}=(0)$. Let $\mathscr{U}$ be any semisimple bialgebra.

\section{REFERENCES}

[B] George M. Bergman, The diamond lemma for ring theory, Adv. in Math. 29 (1978), 178-218.

[GNT] J. A. Green, W. Nichols and E. J. Taft, Left Hopf algebras, preprint.

[HR] R. G. Heyneman and D. E. Radford, Reflexivity and coalgebras of finite type, J. Algebra 28 (1974), 215-246.

[HS] R. G. Heyneman and M. E. Sweedler, Affine Hopf algebras I, J. Algebra 13 (1969), 192-241.

[N] W. Nichols, Quotients of Hopf algebras, Comm. Algebra 6 (1978), 1789-1800.

[S] M. E. Sweedler, Hopf algebras, Benjamin, New York, 1969.

[T] M. Takeuchi, Free Hopf algebras generated by coalgebras, J. Math. Soc. Japan 23 (1971), 561-582.

Department of Mathematics, University of Illnnois at Chicago Circle, Chicago, Ilunois 60680

Current address: Department of Mathematics, Rutgers University, New Brunswick, New Jersey 08903 